\title{
An Automatic Evaluation System for the Photometric Performance of Vehicle Headlamps Using Image Processing Algorithms
}

\author{
Yandan Lin*,Yuan Gao, Yaojie Sun \\ Department of Illuminating Engineering and Light Sources \\ Fudan University \\ Shanghai, China \\ *ydlin@fudan.edu.cn
}

\author{
Shijun Zhang, Wei Wang \\ The Institute of Motor Vehicle Lamp Inspection \\ Shanghai Motor Vehicle Inspection Center \\ Shanghai, China
}

\begin{abstract}
Global report reveals that about 1.24 million road traffic deaths occur annually on the world's roads. The beam pattern is an important characteristic of the headlamp for road safety. Measurement by traditional testing methods using a CIE A-a type goniophotometer cost enormous amount of time and money. In this study, an automatic evaluation method based on image processing is proposed for saving testing time and space. The novel concept of I-matrix is applied in the evaluation algorithm. Comparison of six different algorithms for edge detection of the cut-off line is shown in this study and the Canny algorithm stands out with an expected result. Experiment results show that the proposed method can export a reliable report with a tolerant error. The automatic alignment procedure based on edge detection algorithms can not only serve this evaluation system, but also be used to replace the visual task for cut-off line aiming in traditional methods.
\end{abstract}

Keywords- illuminance distribution measurement; passing beam assessment; image processing; cut-off line aiming; edge detection

\section{INTRODUCTION}

According to the global status report on road safety 2013 by the World Health Organization (WHO), about 1.24 million road traffic deaths occur annually on the world's roads since 2007 [1]. Meanwhile, the number of registered vehicles increases $15 \%$ globally [1]. Besides related laws, the enhanced safety quality of vehicles and roads will help reduce the traffic accident rate. Especially at night, the proper lighting equipment can contribute to improving visibility and detection range for road users and thus reducing traffic accidents and fatalities.

As vehicles evolve, so do automotive lighting and corresponding standards for road safety. Currently, there are various administrative provisions on vehicle headlamps, such as ECE and SAE, and China developed its own national standards GB after ECE. All the provisions and standards should guarantee that the headlamps shall be so made that they give adequate road illumination and protect against excessive glare when emitting the passing beam $[2,3]$. The illuminance distribution of the principal passing beam headlamp shall incorporate a "cut-off" (see Fig .1), which enables the headlamp to be adjusted correctly for the photometric measurements and for the aiming on the vehicle [2].
Nowadays, most of testing agencies usually use a CIE A- $\alpha$ type goniophotometer (shown in Fig .2) to test a headlamp sample with the test sample rotating around both horizontal axis and vertical axis, and then the designed software can calculate target illuminance distribution. The whole testing system, however, is quite expensive for average companies. What's more, the test is not online. Every test will cost the client a long time and a great deal of money.

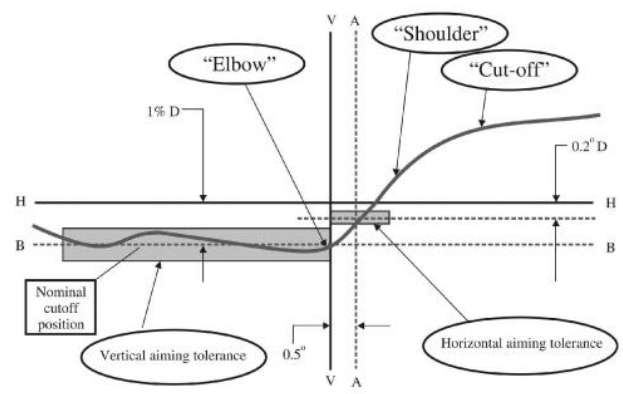

Figure 1. A typical cut-off line described in ECE Regulation.

Since the standard headlamp testing should be carried out in a darkroom with the length of $25 \mathrm{~m}$ at least, it is quite a high requirement for research institutions or manufacturers, with a considerable cost of test field, especially in big cities like Shanghai. In this case, near-field optics technologies are proposed by institutions around the world for the assessment of car headlamp beam geometric and photometric properties. The standard testing length of $25 \mathrm{~m}$ is reduced to $3-5 \mathrm{~m}$ through optical transformation [4-7] or photometric calculations [8, 9]. The illuminance distribution of the vertical screen for headlamp assessment is obtained by two basic approaches: 1) analyzing the image projected by the headlamp on a near-field screen [4, 7-9] and 2) detecting the intensity and direction of the headlamp directly using a radiometric CCD camera [5, 6]. Pervious research has proved that luminance and illuminance can be measured by a digital camera $[10,11]$. With respect to the cut-off line detection and aiming, a simple method of identifying the dark-light border by searching the maximum of the normalized gradient of the column's profile with a mean filter was mentioned in [4]. Previous research on the subject of 
cut-off line detection does not incorporate the variety of algorithms examined in this study.

In this paper, we propose a fast testing method for the low beam of headlamp based on image processing algorithms. Firstly, a brief introduction of GB 4599-2007 was made for a better understanding of testing requirements. Then, the novel concept of the I-matrix was induced to the headlamp assessment, making the theory of matrix and image processing available for the illuminance calculation. According to different site requirements, $25 \mathrm{~m}$ and $4 \mathrm{~m}$ methods were proposed and relevant experiments were conducted. Results showed that the fast method proposed in this study could export the same accurate report as traditional methods, but with less time and space consumption.

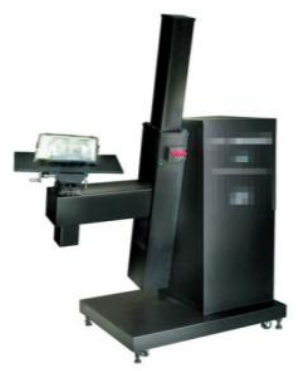

Figure 2. A CIE A- $\alpha$ type goniophotometer.

\section{AVAILABLE STANDARDS}

According to GB 4599-2007, the illumination produced by the headlamp shall be determined by means of a flat vertical screen set up $25 \mathrm{~m}$ forward of the headlamp, at right angles to its axes as shown in Fig .3. The test screen shall be sufficiently wide to allow examination and adjustment of the "cut-off" of the passing beam over at least $5^{\circ}$ on either side of the $\mathrm{V}-\mathrm{V}$ line.

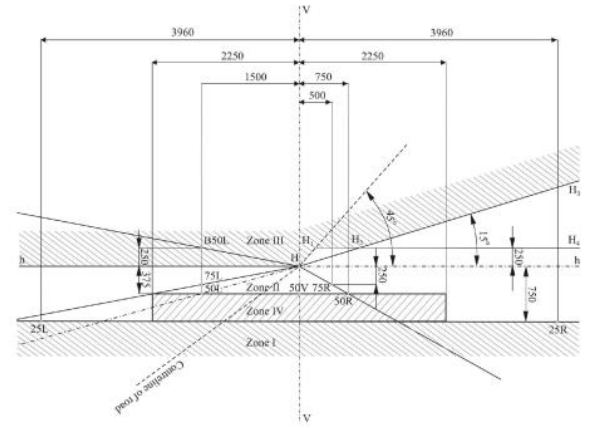

Figure 3. Testing points and zones of headlamp's passing beam for right-hand traffic (dimension in $\mathrm{mm}$ with screen at $25 \mathrm{~m}$ distance).

The illumination produced on the screen by the passing beam shall meet the requirements as shown in Table I.

TABLE I. TABLE TYPE STYLES

\begin{tabular}{|c|c|c|}
\hline Point on measuring screen & \multicolumn{2}{|c|}{ Required illumination in lux } \\
\hline Point B 50 L & $\leq 0.4$ & $\leq 0.4$ \\
\hline Point 75 R & $\geq 6$ & $\geq 12$ \\
\hline Point 75 L & $\leq 12$ & $\leq 12$ \\
\hline Point 50 L & $\leq 15$ & $\leq 15$ \\
\hline Point 50 R & $\geq 6$ & $\geq 12$ \\
\hline Point 50 V & - & $\geq 6$ \\
\hline
\end{tabular}

\begin{tabular}{|c|c|c|}
\hline Point 25 L & $\geq 1.5$ & $\geq 2$ \\
\hline Point 25 R & $\geq 1.5$ & $\geq 2$ \\
\hline Any point in zone III & $\leq 0.7$ & $\leq 0.7$ \\
\hline Any point in zone IV & $\geq 2$ & $\geq 3$ \\
\hline Any point in zone I & $\leq 20$ & $\leq 2 \mathrm{E}^{*}$ \\
\hline$* E$ is the actually measured value in points 50 R respectively $50 \mathrm{~L}$ \\
\hline
\end{tabular}

The illumination values in zones " $\mathrm{A}$ " and " $\mathrm{B}$ " as shown in Fig .4 shall be checked by the measurement of the photometric values of points 1 to 8 on this figure; these values shall lie within the following limits:

$$
\begin{aligned}
& 1+2+3 \geqslant 0.3 \operatorname{lux}, \text { and } \\
& 4+5+6 \geqslant 0.6 \operatorname{lux}, \text { and } \\
& 0.7 \operatorname{lux} \geqslant 7 \geqslant 0.1 \text { lux and } \\
& 0.7 \operatorname{lux} \geqslant 8 \geqslant 0.2 \operatorname{lux}
\end{aligned}
$$

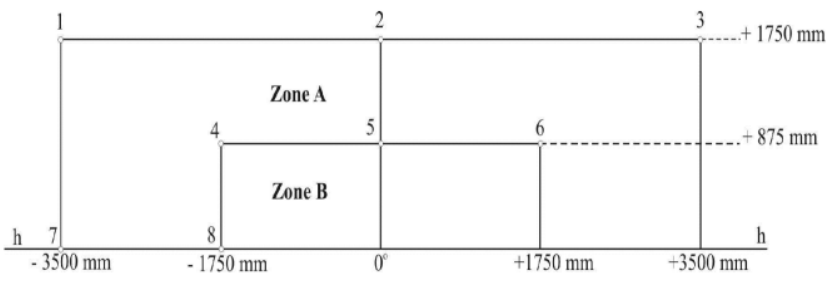

Figure 4. Testing zones A and B.

Obviously, the standard assessment requires not only the illuminance values of certain points on the screen, but also the illuminance distribution of specific zones. Instead of the traditional method achieved by rotating and scanning the headlamp point by point, the fast measurement method proposed in this study introduces a novel concept of I-matrix for effective algorithm implementation. Definition and parameters of this concept is presented in detail in the next section.

\section{A NEW CONCEPT APPLIED IN AUTOMOTIVE LIGHTING MEASUREMENT}

In the area of photometric measurement, the illuminance information of the target working plane is usually described with the average illuminance $(\bar{E})$ and the uniformity ratio of illuminance (R), sometimes presented in the form of a matrix, such as in the procedure of road illuminance measurement. The mathematic expression of the matrix delivers more light distribution messages than $\bar{E}$ and R. However, the exact definition of the illuminance matrix is not available until the novel concept of the I-matrix is proposed in [12]. The original purpose of the I-matrix concept is to achieve precise intelligent lighting control. But further research shows that it is also suitable for illuminance measurement.

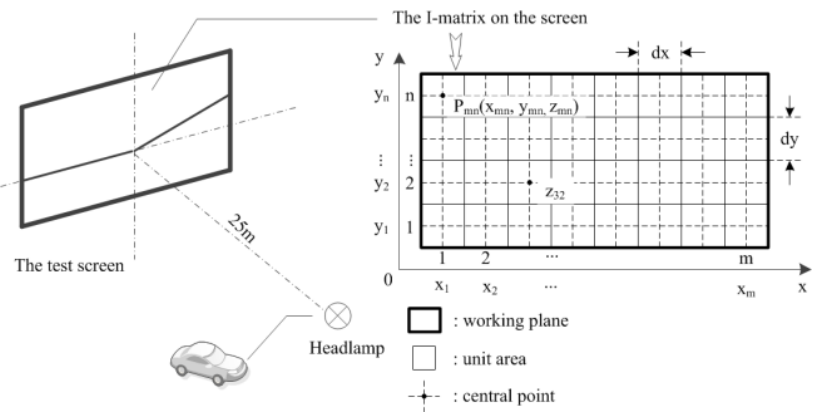

Figure 5. The definition of the I-matrix. 
As shown in Fig .5, the test screen is illuminated by the passing beam from the aimed headlamp. As the target working plane, the screen should be divided into $m \times n$ nearly square areas, which should be small enough to meet the accuracy requirement. Then the I-matrix of the plane is established with each central point illuminance of the unit area as the element of the matrix. $P(x, y, E)$ is an arbitrary point on the screen, where $(x, y)$ shows the position of $P$, and $E$ is the point illuminance value of $P . d x$ and $d y$ are the side length of the unit area respectively, and they can be easily calculated by

$$
\begin{aligned}
& d x=\frac{x_{m}-x_{1}}{m-1}, \\
& d y=\frac{y_{n}-y_{1}}{n-1} .
\end{aligned}
$$

Then a derived concept is defined as the I-matrix density (ID), which can be calculated by the inverse of the unit area, i.e.,

$$
I D=\frac{1}{d x \times d y} .
$$

Basically, ID is positive correlated to the accuracy of the Imatrix.

Though the form of illuminance written in a matrix has been exciting for long in photometric measurement, relevant concept was rarely mentioned because people took it for granted. The novel concept of I-matrix is suitable for digital signal processing due to the powerful matrix theory. Moreover, the I-matrix perfectly matches the data storage form of a digital image, which is the foundational technology for the rapid measurement of illuminance distribution.

\section{I-MATRIX ACQUISITION BASED ON IMAGE PROCESSING}

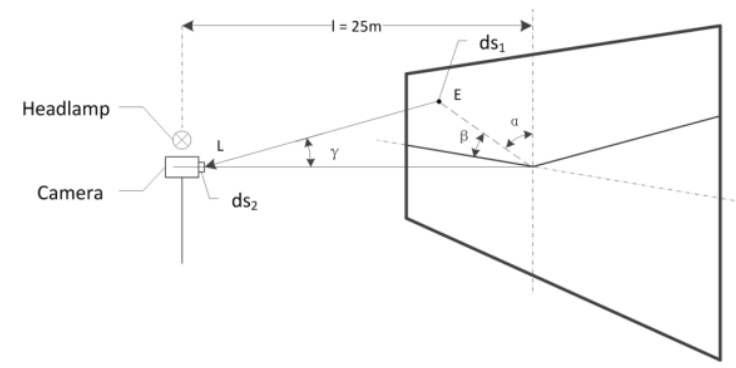

Figure 6. The basic principle of rapid measurement in this study.

It has been proved that the luminance and illuminance can be measured and calculated by a digital camera [10, 11]. The basic principle of rapid measurement in this study is demonstrated as below.

$\mathrm{E}$ is the illuminance value of an arbitrary point on the screen $25 \mathrm{~m}$ away from the headlamp and camera. $\mathrm{M}$, the luminous exitance value of this point, is given by

$$
M=\rho \cdot E,
$$

where $\rho$ is the reflectance of this point. If the surface of the screen is diffuse (Lambertian), the surface luminance is independent of the observation angle, given by

$$
L=M / \pi \text {. }
$$

Even if the screen is not a perfect Lambert surface, L could still be seen as an angle-independent parameter since $l$ is sufficient long that the angle $\gamma$ could be ignored. Additionally, the NTSC luminance $\mathrm{Y}$ of certain point on the image is an uncorrected luminance value. Therefore, $\mathrm{Y}$ can be converted to E by performing a nonlinear calibration $\mathrm{C}(\mathrm{Y})$.

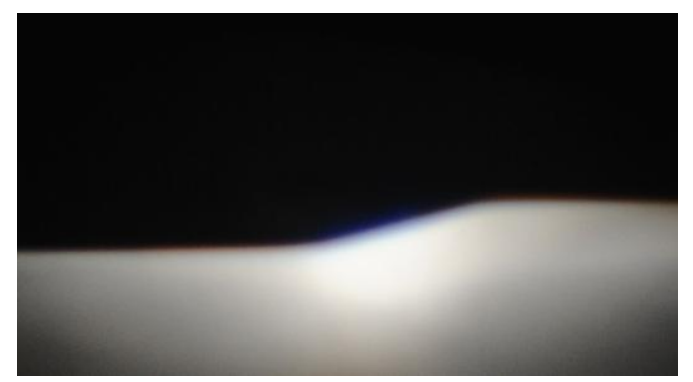

Figure 7. A digital image of light distribution on the screen taken by a camera.

In order to get $\mathrm{C}(\mathrm{Y})$, a pilot experiment was conducted by taking a picture with a digital camera (NIKON D700, f/5.6, 1/8 s), as shown in Fig .7. Then, traditional measurement was taken to get a precise I-matrix. By comparing the Y-matrix from the digital image with the I-matrix, Y-I fitting curve was gained after a running an average filter, as shown in Fig .8. With this curve, we can easily obtain the I-matrix by processing a digital image.

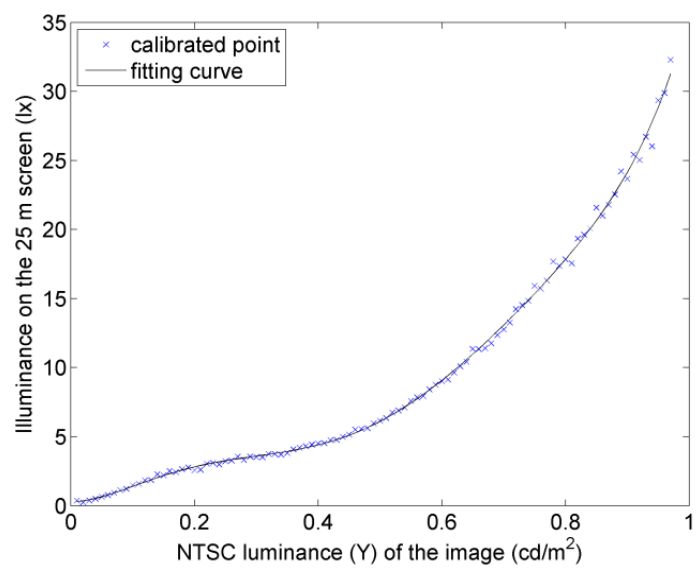

Figure 8. The Y-I fitting curve.

Though the method mentioned above can achieve a rapid measurement of illuminance distribution for headlamp assessment, it still requires a large space for the testing operation. Therefore, as shown in Fig .9, we proposed a fast measurement method which just requires $4 \mathrm{~m}$ distance for online testing. The calibration procedure, however, seems a little more complex than that of $25 \mathrm{~m}$ method. Since the distance between light source and the screen becomes shorter, the impact of angle can no longer be ignored. In another word, each point of the screen holds an individual nonlinear 
calibration curve. As the fitting curve stems from discrete $\mathrm{E}$ and $\mathrm{Y}$ data, the curve could be deemed as a $k \times 2$ matrix. Then the $m \times n$ I-matrix on the screen requires a $m \times n \times k \times 2$ calibration matrix, i.e., $C_{\text {matrix }}$. The procedure of gaining a $C_{\text {matrix }}$ and the I-matrix is listed as below.

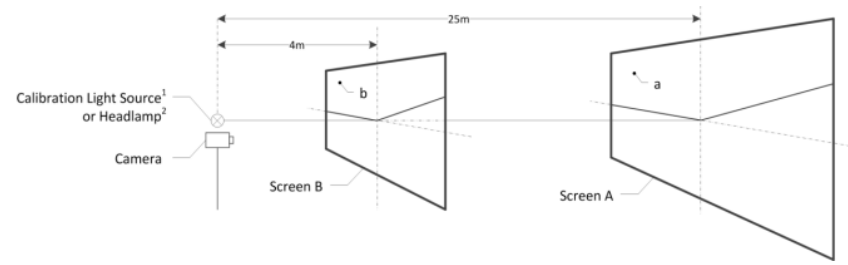

Figure 9. A fast measurement method which just requires 4 m distance.

Step 1: Choose Screen A and calibration light source, which is dimmable from $L_{1}$ to $L_{k}$. Obtain the $I_{\text {matrix } 25 m}$ on Screen A projected by calibration light source (with the luminance of $L_{1}$ ) through traditional methods of headlamp measurement;

Step 2: Choose Screen B and calibration light source. Take a picture of the light distribution on Screen B projected by calibration light source (with the luminance of $L_{1}$ ) with a digital camera;

Step 3: Change the luminance of calibration light source and repeat Step $1 \& 2$;

Step 4: $C_{\text {matrix }}$ is formed referring to Table II;

TABLE II. REQUIRED PARAMETERS FOR $C_{\text {matrix }}$

\begin{tabular}{|c|c|c|}
\hline $\begin{array}{l}\text { The luminance of } \\
\text { calibration light source }\end{array}$ & $\begin{array}{c}\text { The I-matrix on } \\
\text { Screen A }\end{array}$ & $\begin{array}{c}\text { The digital image of } \\
\text { Screen B }\end{array}$ \\
\hline$L_{l}$ & $I_{\text {matrix } 25 m_{2} I}$ & $Y_{\text {matrix } 4 m_{1} 1}$ \\
\hline$L_{2}$ & $I_{\text {matrix_25m_2 }}$ & $Y_{\text {matrix } \_4 m \_2}$ \\
\hline$\ldots$ & $\ldots$ & $\ldots$ \\
\hline$L_{k}$ & $I_{\text {matrix_25m_k }}$ & $Y_{\text {matrix_4m_k }}$ \\
\hline
\end{tabular}

Step 5: Choose Screen B and headlamp. Take a picture of the light distribution on Screen B projected by headlamp with a digital camera (the same position, aperture, exposure time, etc. as Step 2 \& 3);

Step 6: Calculate the I-matrix with the $C_{\text {matrix }}$.

It is notable that interpolation algorithms should be implemented if required point is not found on the $C_{\text {matrix }}$. Moreover, once the $C_{\text {matrix }}$ is gained, Step 1-4 can be skipped for the following rapid measurement of headlamps. However, the automatic assessment of headlamps cannot be achieved by just gaining the I-matrix, because the corresponding position of the I-matrix on the $25 \mathrm{~m}$ screen is not clear. Hence, it is necessary to develop effective algorithms to position the Imatrix automatically through cut-off line recognition, which is elaborated in the following section.

\section{CuT-OFF Line ReCOGNITION FOR Automatic Aiming}

Since a requirement for correct aiming of low-beam visual cut-off line is specified within existing regulations, automatic aiming becomes an important part of the fast method for headlamp assessment. The aiming algorithms will not only lead to an accurate $C_{\text {matrix }}$ or calibration curve in the calibration procedure, but also replace the visual aiming for error reduction in traditional methods.

The CIE 183 document recommended Pollack model as the cut-off line model [13]. The visual recognition of a cut-off in a light pattern is deemed as the reaction of the human eye to the contrast between neighboring parts of the screen where the luminance shows a significant variation. Luminance differences are only visible if they are greater than a certain threshold given as being the smallest difference of apparent luminance discriminated by the human eye. His model, called ATN (apparent threshold number), can be expressed as

$$
A T N=4.91 \frac{E_{2}-E_{1}}{\left(E_{1}+E_{2}\right)^{0.63}},
$$

in which $E_{1}$ and $E_{2}$ are the illuminances of two points on the screen which have an angular distance of 0.05 degree. With this definition, cut-off line can be easily found with a known Imatrix. Mostly, however, we need to aim the cut-off line before we obtain the I-matrix.

Since the fast measurement method proposed in this study is based on image processing technology, the headlamp alignment procedure can also be implemented through classic image segmentation algorithms. As part of the segmentation process, edge detection is one of the most commonly used operations in image analysis [14]. In this study, experiments of image edge detection were conducted with six different algorithms (Sobel, Prewitt, Roberts, Log, Zerocross, and Canny method) [15]. Results shown in Fig .10 illustrate that the Canny edge detector revealed outstanding performance of noise immunity and directional research as the outcome edge is nearly a continuous line. Cut-off lines defined by Pollack model (red line), and detected by Canny algorithm (blue) are overlaid on the light distribution image to prove the feasibility of the edge detection method. It could be seen from Fig .11 that the Canny algorithm matched perfectly with the image, but the Pollack model appeared a sawtooth waveform around the elbow and shoulder area. Therefore, Canny edge detection algorithm is determined as the default method in the following study. 


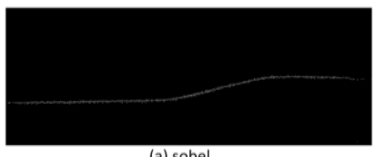

(a) sobel

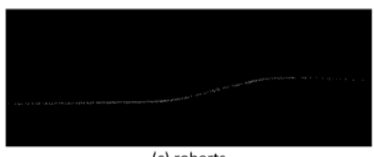

(c) roberts

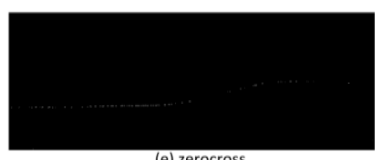

(e) zerocross

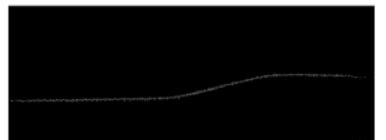

(b) prewitt
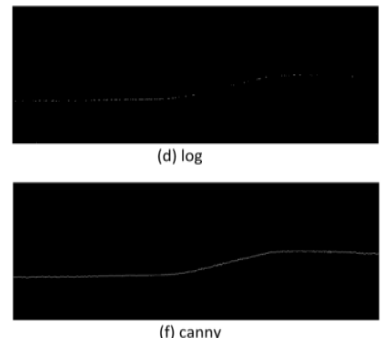

(f) canny
Figure 10. Comparison of image edge detection with six different algorithms.

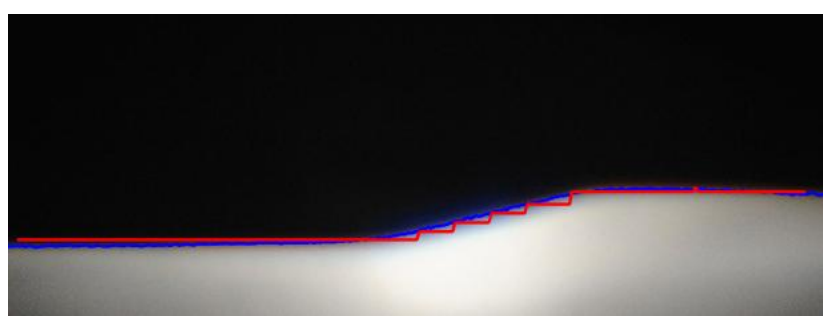

Figure 11. Cut-off lines defined by Pollack model (red line), and detected by Canny algorithm (blue) are overlaid on the light distribution image.

\section{RESULTS}

Experiments on the fast measurement using the screen $25 \mathrm{~m}$ away were conducted to verify our hypothesis. The converted I-matrix is obtained from the digital image by camera using the calibration curve. Contour maps of the standard and converted I-matrix are compared in Fig .12. It is obvious that they contain the similar distribution, but a little shift and discontinuity can be found in the converted I-matrix. This might be due to the inevitable deviation in the headlamp alignment procedure and the noise on the digital image.
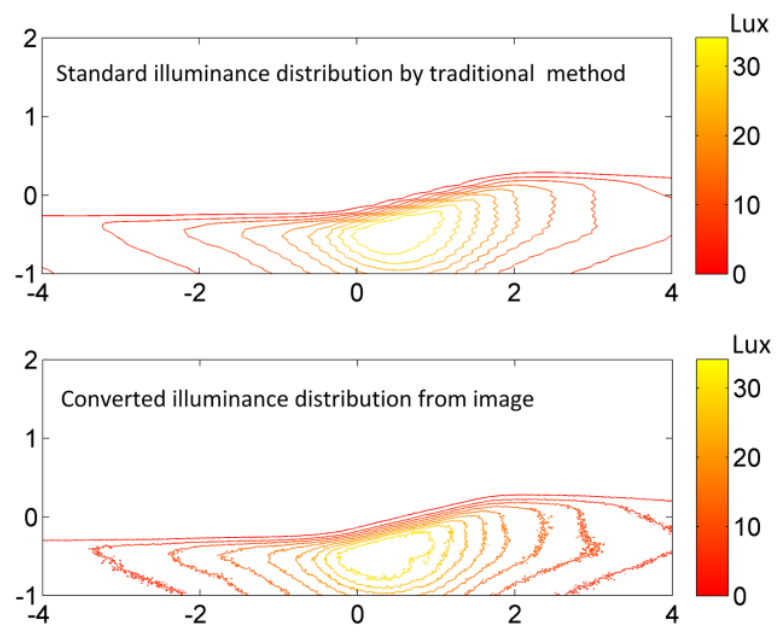

Figure 12. Contour maps of the standard and converted I-matrix.

20 points emphasized in the GB standard are chosen for error analysis. As shown in Fig .13, the logarithmic coordinate of illuminance values is used for better observation. It can be concluded that the deviation can be controlled within $\pm 20 \%$ for most points. With respect to small illuminance values, the deviation will raise to over $70 \%$. This may be caused by the inaccurate part of low illuminance in calibration curve, which is formed with the same interval for all illuminance levels. But it has limited impact on the testing results regulated in the GB standard. Experimental results show that the converted I-matrix exports the same report as the standard I-matrix does. Therefore, this method is suitable for online headlamp development and rapid testing.

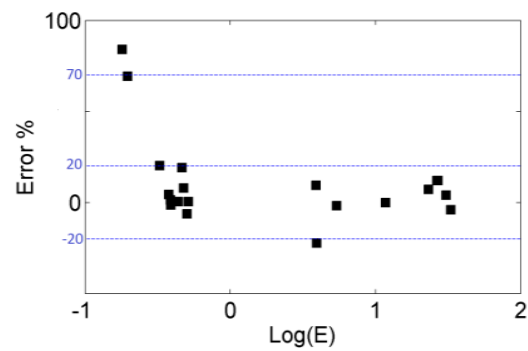

Figure 13. Error distribution.

\section{CONCLUSIONS}

In this study, a rapid testing method for headlamp light distribution using a camera is proposed and the $25 \mathrm{~m}$ method has been verified. The test for $4 \mathrm{~m}$ method, however, is in the planning because of the high requirement of calibration light sources and diffused screen. The accomplished experiments show that this method is feasible with a tolerable error. The basic flow of the fast testing method within $4 \mathrm{~m}$ is presented in Fig. 14.

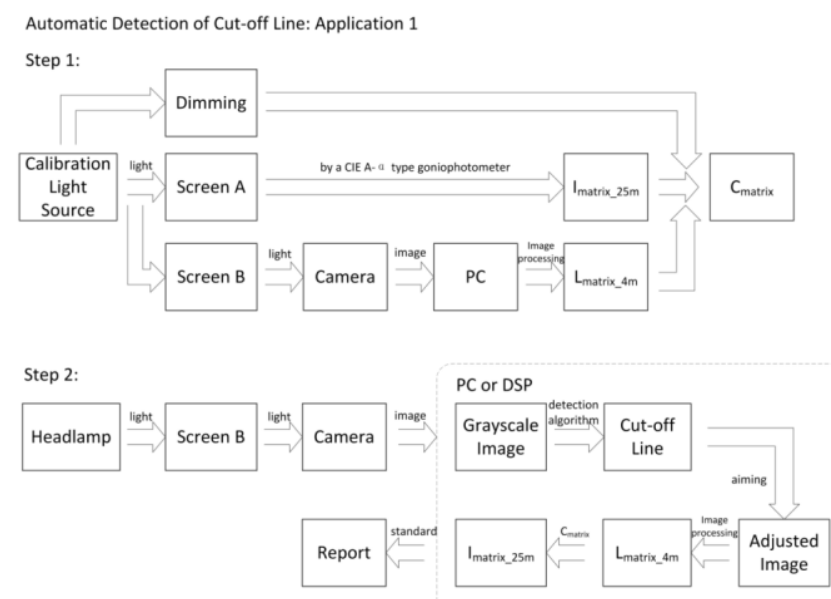

Figure 14. The flow chart of the $4 \mathrm{~m}$ method.

The headlamp alignment procedure through image edge detection methods is also applicable to traditional methods for headlamp testing. The flow chart in Fig .15 reveals that the common visual alignment task is replaced with a precise control system with suitable edge detection algorithms. This design will avoid the inaccurate operator-dependent alignment and thus improve the subsequent assessment accuracy of the headlamp's beam. 


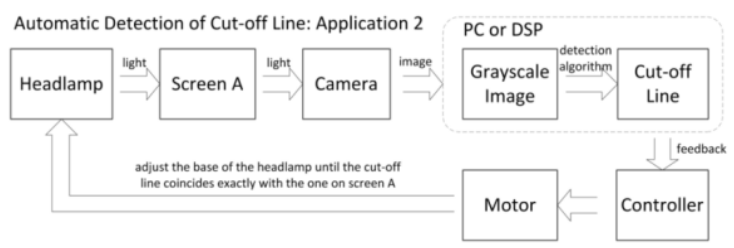

Figure 15. The flow chart of a traditional method with an automatic alignment procedure using edge detection algorithms.

\section{DISCUSSION AND FURTHER WORK}

It is believed that the headlamp testing will be conducted in a more convenient and intelligent way in the future. The method proposed in this study presents an excellent performance on the aiming and testing speed compared with the traditional method with a goniophotometer. From a digital image, localized details can be easily detected which might be missed the rotating goniophotometer.

Though the theory has been verified in the laboratory environment, there is a long way to go before a workable prototype is developed. Following tasks should be focused on 1) programmed camera with autoexposure procedure to avoid overexposure, underexposure, or glare, 2) improved edge detection algorithms for all qualities of light shape in the aiming procedure, 3) suitable diffused materials for the nearfield screen and calibration light sources for the $4 \mathrm{~m}$ method.

\section{ACKNOWLEDGMENT}

This work is cooperated with the Institute of Motor Vehicle Lamp Inspection in Shanghai Motor Vehicle Inspection Center. The work is supported by the projects of general administration of quality supervision, inspection and quarantine of science and technology (Project NO. 2013QK145); National Basic Research Program of China (973) (Project NO. 2010CB734102); Science and Technology Commission of Shanghai Municipality (Project NO. 14441900400); National Natural Science Foundation of China (Project No. 61374129).

\section{REFERENCES}

[1] W. H. Organization, WHO global status report on road safety 2013: supporting a decade of action: World Health Organization, 2013.

[2] ECE Regulation No. 112: Uniform provisions concerning the approval of motor vehicle headlamps emitting an asymmetrical passing beam or a driving beam or both and equipped with filament lamps and/or lightemitting diode (LED) modules, 2010.

[3] CIE 188 Technical Report: Vehicle Headlighting Systems Photometric Performance - Method of Assessment, 2010.

[4] A. Bevilacqua, A. Gherardi and L. Carozza, "An automatic system for the real-time characterization of vehicle headlamp beams exploiting image analysis," Instrumentation and Measurement, IEEE Transactions on, vol. 59, pp. 2630-2638, 2010.

[5] S. Royo, M. J. Arranz, J. Arasa, M. Cattoen, and T. Bosch, "New costeffective sensor for the characterization of automotive headlamps by measurements in the near field," Sensors and Actuators A: Physical, vol. 132, pp. 56-62, 2006.

[6] S. Royo, M. J. Ús Arranz, J. Arasa, M. Cattoen, and T. Bosch, "Lowcost photometric testing of automotive headlamps from near-field measurement,".

[7] M. Cattoen, S. Royo, M. J. Arranz, J. Arasa, and H. C. Seat, "Compact system for photometric characterization of automotive headlamps," in Instrumentation and Measurement Technology Conference, 2005. IMTC 2005. Proceedings of the IEEE, 2005, pp. 1669-1674.

[8] W. Wang, S. To, C. Cheung, J. Jiang, and B. Wang, "A new method to test the photometric characteristics of lamps for motor vehicles," OptikInternational Journal for Light and Electron Optics, vol. 120, pp. 549. 552, 2009.

[9] H. P. Wu, Y. Lee and S. Chang, "Fast measurement of automotive headlamps based on high dynamic range imaging," Applied optics, vol. 51, pp. 6870-6880, 2012.

[10] D. Wüller and H. Gabele, "The usage of digital cameras as luminance meters," in Electronic Imaging 2007, 2007, p. 65020U-65020U-11.

[11] M. Moeck and S. Anaokar, "Illuminance analysis from high dynamic range images," Leukos, vol. 2, pp. 211-228, 2006.

[12] Y. Gao, Y. Lin and Y. Sun, "A wireless sensor network based on the novel concept of an I-matrix to achieve high-precision lighting control," Building and Environment, vol. 70, pp. 223-231, 2013.

[13] CIE 183 Technical Report: CIE 183Definition of the Cut-off of Vehicle Headlamps, 2008.

[14] J. R. Parker, Algorithms for image processing and computer vision: John Wiley \& Sons, 2010.

[15] R. Maini and H. Aggarwal, "Study and comparison of various image edge detection techniques," International Journal of Image Processing (IJIP), vol. 3, pp. 1-11, 2009. 